\title{
Prevalence of hepatitis B antiviral drug resistance variants in North American patients with chronic hepatitis B not receiving antiviral treatment
}

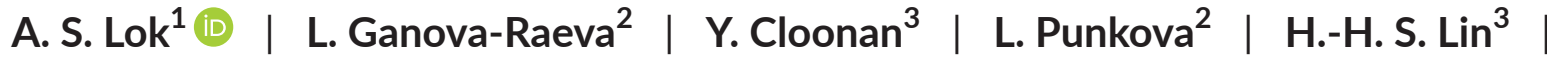 \\ W. M. Lee ${ }^{4}$ | M. G. Ghany ${ }^{5}$ for the Hepatitis B Research Network (HBRN) \\ ${ }^{1}$ Division of Gastroenterology and Hepatology, University of Michigan, Ann Arbor, MI, USA \\ ${ }^{2}$ Centers for Disease Control and Prevention, Division of Viral Hepatitis Laboratory Branch, Atlanta, GA, USA \\ ${ }^{3}$ Department of Epidemiology, University of Pittsburgh, Pittsburgh, PA, USA \\ ${ }^{4}$ University of Texas Southwestern Medical Center, Dallas, TX, USA \\ ${ }^{5}$ Liver Diseases Branch, National Institute of Diabetes, Digestive and Kidney Diseases, National Institutes of Health, Bethesda, MD, USA
}

\section{Correspondence}

Anna Lok, Division of Gastroenterology and Hepatology, University of Michigan, Ann

Arbor, MI, USA.

Email: aslok@umich.edu

\section{Funding information}

The HBRN was funded by a U01 grant from the National Institute of Diabetes and Digestive and Kidney Diseases to the following investigators Lewis R. Roberts, MB, ChB, PhD (DK 082843), Anna SukFong Lok, MD (DK082863), Steven $\mathrm{H}$. Belle, PhD, MScHyg (DK082864), KyongMi Chang, MD (DK082866), Michael W. Fried, MD (DK082867), Adrian M. Di Bisceglie, MD (DK082871), William M. Lee, MD (DK082872), Harry L. A. Janssen, MD, PhD (DK082874), Daryl T-Y Lau, MD, MPH (DK082919), Richard K. Sterling, MD, MSc (DK082923), Steven-Huy B. Han MD (DK082927), Robert C. Carithers, MD (DK082943), Norah A. Terrault, MD, MPH (DK082944), an interagency agreement with NIDDK: Lilia M. Ganova-Raeva, PhD (A-DK-3002-001) and support from the intramural program, NIDDK, NIH: Marc G. Ghany, MD. Additional funding to support this study was provided to Kyong-Mi Chang, $\mathrm{MD}$, the Immunology Center, (NIH/NIDDK Center of Molecular Studies in Digestive and Liver Diseases P30DK50306, NIH Public Health Service Research Grant M01RR00040), Richard K. Sterling, MD, MSc (UL1TR000058, NCATS (National Center for Advancing Translational Sciences, NIH),

\section{Summary}

Antiviral drug resistance hepatitis B virus (HBV) variants (HBV-DR) occur spontaneously in chronic hepatitis $B(C H B)$ patients and after exposure to nucleos $(t) i d e$ analogues (NUCs). We determined the prevalence of HBV-DR variants among participants of the Hepatitis B Research Network (HBRN) Cohort Study conducted at 21 sites in the United States (US) and Canada. Samples obtained from 1342 CHB participants aged $\geq 18$ years, and who were currently not receiving NUCs, were tested for HBV-DR variants by Sanger sequencing. In addition, next generation sequencing (NGS) was used to characterize HBV-DR variants from 66 participants with and 66 participants with no prior NUC exposure matched for HBV genotype and HBV DNA level. Half the participants were men, 75\% Asian, 26\% HBeAg positive. Primary HBV-DR variants were detected by Sanger sequencing in 16 (1.2\%) participants: $2 / 142$ (1.4\%) with and 14/1200 (1.2\%) without prior NUC exposure; only 1 of these 16 had a secondary variant. In total, 23 (1.7\%) participants had secondary variants, including 1 with prior NUC experience. In the subset of 132 participants, NGS detected HBV-DR variants in a higher proportion of participants: primary variants in 18 (13.6\%) (8 [12.1\%] with, and 10 [15.2\%] without prior NUC therapy) and secondary variants in $10(7.6 \%)$ participants. Based on Sanger sequencing, prevalence of primary HBV-DR variants is low (1.2\%) among adults with CHB in US/Canada. The similar low prevalence of HBV-DR variants in participants with and without NUC treatment suggests transmission of these variants is uncommon.

\section{KEYWORDS}

antiviral treatment, hepatitis B virus, next generation sequencing, nucleos(t)ide analogues,

Sanger sequencing

Abbreviations: ALT, alanine aminotransferase; ChB, chronic hepatitis B; $\mathrm{Cl}$, confidence interval; $\mathrm{HBeAg}$, hepatitis Be antigen; HBsAg, hepatitis B surface antigen; $\mathrm{HBV}$, hepatitis B virus; NGS, next generation sequencing; NUC, nucleos(t)ide analogues; PCR, polymerase chain reaction. 
Norah A. Terrault, MD, MPH (CTSA Grant Number UL1TR000004), Michael W. Fried, MD (CTSA Grant Number UL1TR001111), and Anna Suk-Fong Lok (CTSA Grant Number UL1RR024986.) Additional support was provided by Gilead Sciences, Inc. and Roche Molecular Systems via a CRADA through the NIDDK.

\section{1 | INTRODUCTION}

Chronic hepatitis B virus (HBV) infection remains a major global health problem with most African countries and many Asian countries still classified as highly endemic areas. ${ }^{1}$ Treatment options for chronic HBV infection include either peginterferon alfa administered for a finite duration or nucleos(t)ide analogues (NUCs) administered for several years and often for life. A disadvantage of long-term NUC administration is the selection for HBV variants that confer resistance to these drugs. These variants are termed primary drug resistance (DR) variants and are more commonly associated with NUCs that have a low genetic barrier to drug resistance such as lamivudine, adefovir and telbivudine than NUCs with a high barrier to drug resistance such as entecavir and tenofovir. HBV-DR variants are selected during NUC treatment because they can overcome suppression by the NUC used but they generally have less replication fitness. ${ }^{2,3}$ However, additional mutations that restore replication fitness may be selected during long-term treatment with NUCs. ${ }^{4}$ These mutations are called secondary or compensatory HBV-DR variants.

Selection of primary HBV-DR variants can lead to virological and biochemical breakthrough, hepatitis flares, hepatic decompensation and even death.

While most HBV-DR variants are selected during NUC therapy, they can also be present in patients with chronic HBV infection who have never been treated with NUCs because HBV replicates through an error prone reverse transcription of pregenomic RNA to HBV DNA. This is further compounded by very high rates of HBV replication of up to $10^{12}$ virions per day in some patients. It has also been suggested that HBV-DR variants may be transmitted from patients receiving NUCs to HBV-susceptible persons. ${ }^{5}$ This is of most concern in countries where NUCs with low barrier to resistance are frequently used as first-line therapy. A meta-analysis of 106 studies examining the prevalence of HBV-DR variants in 12212 persons with chronic HBV infection who had never received NUCs found an overall prevalence of $5.73 \%$ (95\% confidence interval [Cl]: $4.85 \%-6.61 \%$ ); however, there were wide geographical variations ranging from $20 \%$ in Africa to $0 \%$ in Brazil. ${ }^{6}$ An important reason for the wide range in results is differences in sensitivities of assays used to detect DR variants. Sensitivities of assays used in these studies range from Sanger or population sequencing which can only detect variants present in $\geq 20 \%$ of the virus pool, line probe (reverse hybridization assay) or mass spectrometry which can detect variants present in roughly $5 \%$ of the virus pool, to next generation sequencing (NGS) which can detect variants present in $<1 \%$ of the virus pool. ${ }^{3,7}$
The finding of HBV-DR variants, particularly primary variants, in patients with chronic HBV infection who have not been treated with NUCs has important epidemiologic and clinical implications. Epidemiologically, the identification of primary variants in NUC-naïve patients would confirm that transmission of these less fit variants can result in chronic infection in susceptible persons. Clinically, this finding indicates that infected patients would be less likely to respond to NUCs that are ineffective against these variants. We took advantage of the large cohort of participants with chronic HBV infection in the Hepatitis B Research Network (HBRN) to 1 determine the prevalence of primary and secondary HBV-DR variants in North American adults with chronic HBV infection not receiving NUC using Sanger sequencing, 2 describe the prevalence of HBV-DR variants according to sociodemographic and clinical characteristics, and 3 compare the findings of Sanger sequencing with NGS in the detection of these variants in a subset of HBRN participants.

\section{2 | METHODS}

\section{1 | Study population}

The HBRN is a cooperative network of investigators funded by the National Institute of Diabetes and Digestive and Kidney Diseases (NIDDK), National Institutes of Health (NIH). The Adult Cohort Study conducted in 21 clinical centres across the United States (US) and in Toronto, Canada, enrolled hepatitis B surface antigen ( $\mathrm{HBsAg}$ )positive adults who were 18 years or older without evidence of hepatic decompensation, hepatocellular carcinoma, liver transplant or human immunodeficiency virus infection and were not receiving antiviral therapy. Participants who met clinical indications for treatment after enrolment into the HBRN Cohort Study received treatment through HBRN clinical trials or standard of care per site investigator. Details of the HBRN Cohort Study protocol and characteristics of the adult participants had been previously described. ${ }^{8}$ Institutional review board or ethics committee approval was obtained from all clinical sites and all participants provided written informed consent.

\subsection{1 | NGS subpopulation}

To compare the detection rate of HBV-DR variants by Sanger sequencing vs NGS, samples from participants who had prior exposure to NUCs for $\geq 90$ days and had available results for HBV-DR variants by Sanger sequencing, and samples from an equal number of participants with no prior exposure to NUC matched for HBV genotype and 
HBV DNA level, were selected for testing of HBV-DR variants by NGS. Selection of samples for NGS occurred in April 2015 while data for Sanger sequencing continued to accrue until July 2016.

\subsection{Data and sample collection}

History of prior HBV antiviral therapy including names of medications, and start and stop dates were based on self-reporting by participants and systematically collected. Research blood samples were collected at each visit and sent to the NIDDK central repository where they were stored at $-80^{\circ} \mathrm{C}$. Research samples collected the baseline visit were used for HBV-DR variant testing; however, when samples were not available at the baseline visit, samples obtained at or prior to the week 24 follow-up visit were used. Clinical laboratory results closest to the research sample collection date were analysed, provided that the results were obtained $>90$ days from any NUC exposure.

\section{3 | Definition of HBV-DR variants}

To focus on variants that have been confirmed to be associated with resistance to HBV NUCs and to avoid the inclusion of polymorphic variants, we reviewed the published literature and defined a priori a list of primary (A181T/V, T184A/C/F/G/I/L/M/S, S202C/G/I, M204I/V, N236T, M250I/L/V) and secondary (L80I/V, I169T, V173L, L180M) HBV-DR variants for analysis in this study.

\section{4 | Laboratory testing}

\section{\begin{tabular}{l|l}
2.4 .1 & 2.4.1 HBV DNA
\end{tabular}}

HBV DNA levels were tested at a Central Virology Laboratory (University of Washington, Seattle, WA, USA) using a real-time polymerase chain reaction (PCR) assay (COBAS Ampliprep/COBAS TaqMan HBV Test, v.2.0; Roche Molecular Diagnostics, Branchburg, NJ, USA), which has a lower limit of detection of $20 \mathrm{lU} / \mathrm{mL}$, and supplemented with local results when research samples were not available for central testing.

\subsection{2 | HBV Genotype}

HBV genotyping was determined at the Molecular Epidemiology and Bioinformatics Laboratory in the Division of Viral Hepatitis at the Centers for Disease Control and Prevention (CDC) with mass spectrometry.

\subsection{3 | HBV-DR variant}

HBV-DR variant testing was performed at the same CDC laboratory. The sequences of the HBV polymerase spanning nucleotide positions 311-1021 were determined by Sanger sequencing. Variants were reported if they comprised more than $25 \%$ of the viral population, based on the height of a secondary chromatogram peak of the consensus sequence. Next generation sequencing was performed on
MiSeq Illumina platform using the v3 (600) chemistry (Illumina, San Diego, CA, USA) that provides paired reads with 301 nucleotides in each direction. The covered region corresponded to nucleotide positions 1225-1935 of the HBV polymerase gene or from amino acid 61 to 257 of the RT domain. To prepare the libraries for each participant, a shorter nested PCR fragment was obtained and tagged with the larger external fragment to ensure complete coverage. The individual samples were barcoded with six specific 10-mer sequences on each end. The barcoded amplicons were indexed for multiplexing with 21 different 6-mer barcodes. Next generation sequencing data were demultiplexed by MiSeq by indexes and then by barcodes on CLC Genomics Workbench v7.5.1. (QIAGEN, Aarhus A/S, www.clcbio. com). Sequence reads with correct barcodes were mapped to reference coding HBV polymerase sequences from the appropriate genotype and analysed for low frequency variants with a cut-off at $\geq 1 \%$, where the frequency is calculated based on the coverage per site. The data for the mutation sites of interest were extracted and compared to the data from Sanger sequencing.

\section{5 | Statistical analyses}

Summary statistics were presented for the overall sample. Demographic, clinical and virological characteristics were described using frequency and percent for categorical variables and median (interquartile range) for continuous variables. The frequency and percent of primary DR variants was given for the overall sample; according to demographic, clinical and virological characteristics; and according to past NUC exposure. Summary statistics were also presented for the NGS subset (overall and by prior NUC use). To compare the distribution of continuous variables between those with and without prior NUC use, Kruskal-Wallis nonparametric tests were performed. Pearson's chi-square tests were used for categorical variables. Statistical significance was determined as $P<.05$. For the subpopulation with at least one DR variant (based on either Sanger or NGS results), we provided individual-level listings to show prior NUC use, HBV genotype, HBV DNA level and both Sanger and NGS results. SAS 9.3 (SAS Institute, Cary NC, USA) was used for statistical analyses.

\section{3 | RESULTS}

\subsection{Characteristics of the study population}

As of 5 July 2016, 1993 participants were enrolled into the HBRN Cohort Study. For this analysis, 430 participants were excluded because they were found to be either HBsAg negative at enrolment or belonged to groups that were specifically targeted for enrolment (participants with acute hepatitis B, flares of chronic hepatitis B (CHB), or hepatitis $D$ coinfection; participants who were pregnant, or were potential candidates for HBRN clinical trials or immunology study). ${ }^{8}$ Figure 1 shows a flow diagram of participant selection. Of 1563 potentially eligible participants, an additional 221 participants were excluded because DR variant testing from research blood samples collected at or before the week 24 visit was not available, HBV DNA 


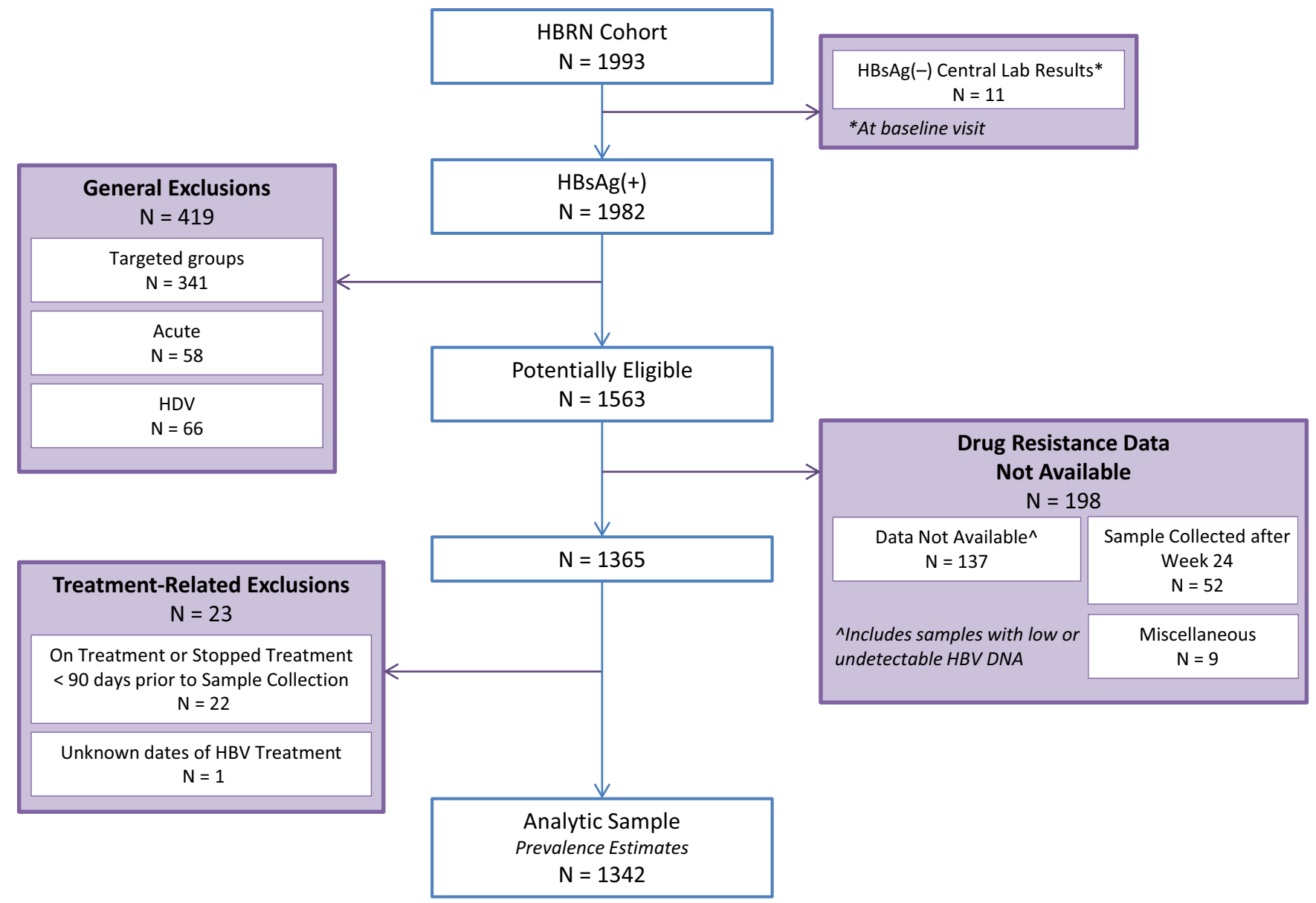

FIGURE 1 Flow diagram of participant selection. Among 1993 participants enrolled, 11 were excluded because they were found to be HBsAg negative at enrolment based on testing at the central laboratory and 419 were excluded because they had acute HBV or HDV infection or belong to special groups enrolled after the protocol was switched from "consecutive" to "targeted" enrolment. Of the 1563 potentially eligible participants, 198 were excluded because samples within the first 24 weeks of enrolment were not available or HBV-DR resistance data were not available (mostly due to low viremia precluding sequencing). An additional 23 participants were excluded because research samples were collected after the participants started treatment or within 90 days of stopping treatment or dates of HBV treatment were unknown. Thus, 1342 participants were included in the analysis.

level was too low for HBV-DR testing, antiviral therapy was stopped less than 90 days prior to research sample collection or dates of HBV antiviral therapy were unknown.

In total, 1342 participants were included in the analysis of prevalence of and risk factors associated with HBV-DR variants based on Sanger sequencing. All 1342 participants included were enrolled prior to switching from "consecutive" to "targeted" enrolment, and all samples used in this study were collected between 2011 and 2014. Median age of the participants studied was 42 years, and $51 \%$ were men. A majority of the participants were Asian (75\%), born outside the USA or Canada (82\%). Approximately one-quarter (26\%) were hepatitis $\mathrm{B}$ e antigen ( $\mathrm{HBeAg}$ ) positive, median HBV DNA level was $4.0 \log _{10}$ $\mathrm{IU} / \mathrm{mL}$, and one-third had alanine aminotransferase (ALT) level within normal range ( $\leq 30 \mathrm{U} / \mathrm{L}$ for men and $\leq 20 \mathrm{U} / \mathrm{L}$ for women). The predominant HBV genotypes were B (39\%) and C (33\%) followed by A (18\%) and D (7\%) (Table 1).

One hundred and forty-two (10.6\%) participants had received NUCs previously ranging in duration from 0.5 to 144 months (median
19.2). The total duration of NUC treatment was $<1$ year in $41 \%$, $1-3$ years in $36 \%$, and $>3$ years in $23 \%$ of treated participants. All 142 participants treated with NUCs had stopped treatment $\geq 90$ days prior to DR variant testing with the vast majority (91.4\%) having stopped NUCs $>6$ months before testing. The most common prior NUC was lamivudine $(n=67)$, with similar numbers having previously received adefovir (23), entecavir (28), or tenofovir (20) and very few having received telbivudin (Table 2 ).

\subsection{Prevalence of primary and secondary HBV-DR variants based on Sanger sequencing}

Sixteen (1.2\%) participants had primary HBV-DR variants based on Sanger sequencing including 2/142 (1.4\%) with prior NUC and $14 / 1200$ (1.2\%) with no prior NUC therapy. Both participants with prior NUC had the A181T/V variant detected (1 prior adefovir and 1 prior tenofovir). Among the 14 participants with no prior NUC therapy, 3 had A181T/V, 2 S202C/G/I, 4 M204I/V, and 5 M250I/L/V 
TABLE 1 Frequency of primary HBV-DR variants (based on Sanger Sequencing) by participant characteristics

\begin{tabular}{|c|c|c|c|}
\hline \multirow[b]{2}{*}{ Characteristic } & \multirow[b]{2}{*}{ All (Column \%) } & \multicolumn{2}{|c|}{$\begin{array}{l}\text { Primary HBV-DR Variant } \\
\text { (Row \%) }\end{array}$} \\
\hline & & Present & Absent \\
\hline $\mathrm{N}$ & 1342 & $16(1.2)$ & $1326(98.8)$ \\
\hline Age (y) & $\mathrm{N}=1342$ & & \\
\hline Median (IQR) & $41.9(33.2,51.7)$ & & \\
\hline $18-<40$ & $592(44.1)$ & $6(1.0)$ & $586(99.0)$ \\
\hline $40-<60$ & $612(45.6)$ & $6(1.0)$ & $606(99.0)$ \\
\hline$\geq 60$ & $138(10.3)$ & $4(2.9)$ & $134(97.1)$ \\
\hline Sex & $\mathrm{N}=1342$ & & \\
\hline Male & $688(51.3)$ & $8(1.2)$ & $680(98.8)$ \\
\hline Race & $N=1339$ & & \\
\hline White & $135(10.1)$ & $2(1.5)$ & $133(98.5)$ \\
\hline Black & $168(12.5)$ & $0(0.0)$ & $168(100.0)$ \\
\hline Asian & $1000(74.7)$ & $13(1.3)$ & $987(98.7)$ \\
\hline Other & $36(2.7)$ & $1(2.8)$ & 35 (97.2) \\
\hline Place of Birth & $N=1338$ & & \\
\hline US or Canada & $235(17.6)$ & $0(0.0)$ & $235(100.0)$ \\
\hline $\begin{array}{l}\text { South America } \\
\text { or other North } \\
\text { America }\end{array}$ & $20(1.5)$ & $1(5.0)$ & $19(95.0)$ \\
\hline Europe & $37(2.8)$ & $1(2.7)$ & $36(97.3)$ \\
\hline Asia or Australia & $927(69.3)$ & $14(1.5)$ & $913(98.5)$ \\
\hline Africa & 119 (8.9) & $0(0.0)$ & $119(100.0)$ \\
\hline $\begin{array}{l}\text { Presumed Source } \\
\text { of hepatitis B } \\
\text { virus (HBV) }\end{array}$ & $\mathrm{N}=1342$ & & \\
\hline $\begin{array}{l}\text { Vertical } \\
\text { Transmission }\end{array}$ & $638(47.5)$ & $4(0.6)$ & $634(99.4)$ \\
\hline $\begin{array}{l}\text { Horizontal } \\
\text { Transmission }\end{array}$ & $388(28.9)$ & $4(1.0)$ & $384(99.0)$ \\
\hline Unknown & $316(23.5)$ & $8(2.5)$ & $308(97.5)$ \\
\hline $\begin{array}{l}\text { Hepatitis B e } \\
\text { antigen }\end{array}$ & $N=1301$ & & \\
\hline Negative & $957(73.6)$ & $10(1.0)$ & $947(99.0)$ \\
\hline Positive & $344(26.4)$ & $6(1.7)$ & $338(98.3)$ \\
\hline $\begin{array}{l}\text { HBV DNA }\left(\log _{10}\right. \\
\text { IU } / \mathrm{mL})\end{array}$ & $N=1311$ & & \\
\hline Median (IQR) & $4.0(3.0,6.1)$ & & \\
\hline$<10^{3}$ & $367(28.0)$ & $4(1.1)$ & $363(98.9)$ \\
\hline $10^{3}-<10^{4}$ & $316(24.1)$ & $6(1.9)$ & 310 (98.1) \\
\hline $10^{4}-<10^{7}$ & $366(27.9)$ & $2(0.5)$ & $364(99.5)$ \\
\hline$\geq 10^{7}$ & $262(20.0)$ & $4(1.5)$ & $258(98.5)$ \\
\hline $\begin{array}{l}\text { Alanine ami- } \\
\text { notransferase } x \\
\text { ULN }\end{array}$ & $N=1290$ & & \\
\hline Median (IQR) & $1.30(0.93,2.00)$ & & \\
\hline$\leq 1$ ULN & 407 (31.6) & $2(0.5)$ & 405 (99.5) \\
\hline
\end{tabular}

TABLE 1 (Continued)

\begin{tabular}{|c|c|c|c|}
\hline \multirow[b]{2}{*}{ Characteristic } & \multirow[b]{2}{*}{ All (Column \%) } & \multicolumn{2}{|c|}{$\begin{array}{l}\text { Primary HBV-DR Variant } \\
\text { (Row \%) }\end{array}$} \\
\hline & & Present & Absent \\
\hline$>1-<2$ ULN & $557(43.2)$ & $12(2.2)$ & 545 (97.8) \\
\hline$\geq 2$ ULN & $326(25.3)$ & $2(0.6)$ & 324 (99.4) \\
\hline HBV Genotype & $N=1309$ & & \\
\hline$A$ & $240(18.3)$ & $3(1.3)$ & 237 (98.8) \\
\hline B & $511(39.0)$ & $6(1.2)$ & 505 (98.8) \\
\hline C & $427(32.6)$ & $7(1.6)$ & $420(98.4)$ \\
\hline $\mathrm{D}$ & $95(7.3)$ & $0(0.0)$ & 95 (100.0) \\
\hline $\begin{array}{l}\mathrm{E} / \mathrm{F} / \mathrm{G} / \mathrm{H} / \\
\text { multiple }\end{array}$ & $36(2.8)$ & $0(0.0)$ & $36(100.0)$ \\
\hline $\begin{array}{l}\text { Prior Treatment } \\
\text { with nucleos(t)ide } \\
\text { analogues (NUC) }\end{array}$ & $N=1342$ & & \\
\hline Yes & $142(10.6)$ & $2(1.4)$ & $140(98.6)$ \\
\hline $\begin{array}{l}\text { Months of prior } \\
\text { NUC } \\
\text { Treatment }^{\mathrm{a}}\end{array}$ & $N=140$ & & \\
\hline Median (IQR) & $19.2(10.0,34.2)$ & $\begin{array}{l}30.5(1 \\
60)\end{array}$ & $\begin{array}{c}19.2 \\
(10.0,33.5)\end{array}$ \\
\hline$<1 \mathrm{y}$ & $58(41.4)$ & $1(1.7)$ & $57(98.3)$ \\
\hline 1 to $3 y$ & $50(35.7)$ & $0(0.0)$ & $50(100.0)$ \\
\hline$>3 y$ & $32(22.9)$ & 1 (3.1) & 31 (96.9) \\
\hline $\begin{array}{l}\text { Time since } \\
\text { stopping HBV } \\
\text { NUC Treatment }\end{array}$ & $N=142$ & & \\
\hline $\begin{array}{l}\text { Median (IQR) } \\
\text { months }\end{array}$ & $42.6(15.5,73.3)$ & $\begin{array}{l}18.1 \\
(14.0 \\
22.2)\end{array}$ & $\begin{array}{l}43.0(15.6, \\
73.7)\end{array}$ \\
\hline$<1 \mathrm{y}$ & $26(18.3)$ & $0(0.0)$ & $26(100.0)$ \\
\hline 1 to $3 y$ & $37(26.1)$ & $2(5.4)$ & $35(94.6)$ \\
\hline$>3 y$ & $79(55.6)$ & $0(0.0)$ & 79 (100.0) \\
\hline
\end{tabular}

ULN, upper limit of normal.

Number of nonmissing variables is shown for each variable. $\mathrm{N}(\%)$ for each level of categorical variables is shown. For continuous variables, median $\left(25^{\text {th }}\right.$ percentile, $75^{\text {th }}$ percentile) are presented.

${ }^{a}$ Start date of NUC treatment unknown for two participants.

(Table 2). Only one of the 16 participants with primary HBV-DR variants had a secondary DR variant detected by Sanger sequencing. The proportion of participants with primary HBV-DR variants in each category of demographic and clinical characteristics was similar (Table 1).

Twenty-three (1.7\%) participants had secondary HBV-DR variants including 1 (0.7\%) with prior NUC and 22 (1.8\%) with no prior NUC therapy. The participant with prior NUC received telbivudine and had L80I/V variant detected, but no primary DR variants detected by Sanger sequencing. The L80I/V variant was also the most common secondary DR variant (20/22) detected among those with no prior NUC (Table 2).

In total, 38 (2.8\%) participants had either primary or secondary HBV-DR variants detected by Sanger sequencing. The prevalence of 
TAB LE 2 Prevalence of primary and secondary HBV-DR variants based on Sanger sequencing, by Prior nucleos(t)ide analogues (NUC) Treatment $(\mathrm{N}=1342)$

\begin{tabular}{|c|c|c|c|c|c|c|c|c|c|}
\hline & \multicolumn{2}{|c|}{$\begin{array}{l}\text { Prior NUC } \\
\text { Treatment }\end{array}$} & \multicolumn{7}{|c|}{ Type of Prior NUC Treatment } \\
\hline & No & Yes & Adefovir & Tenofovir & Telbivudine & Lamivudine & Entecavir & Emtricitabine & Unknown \\
\hline $\begin{array}{l}\text { Primary DR variant } \\
\text { Present, } \mathrm{n}(\%)\end{array}$ & $14(1.2)$ & $2(1.4)$ & $1(4.3)$ & $1(5.0)$ & $0(0.0)$ & $0(0.0)$ & $0(0.0)$ & $0(0.0)$ & $0(0.0)$ \\
\hline $\mathrm{S} 202 \mathrm{C} / \mathrm{G} / \mathrm{I}$ & $2(0.2)$ & $0(0.0)$ & 0 & 0 & & & & & \\
\hline M204I/V & $4(0.3)$ & $0(0.0)$ & 0 & 0 & & & & & \\
\hline M250I/L/V & $5(0.4)$ & $0(0.0)$ & 0 & 0 & & & & & \\
\hline $\begin{array}{l}\text { Secondary DR variant } \\
\text { present, } \mathrm{n}(\%)\end{array}$ & $22(1.8)$ & $1(0.7)$ & $0(0.0)$ & $0(0.0)$ & $1(20.0)$ & $0(0.0)$ & $0(0.0)$ & $0(0.0)$ & $0(0.0)$ \\
\hline L80IV & $20(1.7)$ & $1(0.7)$ & & & 1 & & & & \\
\hline
\end{tabular}

Data presented as number and per cent (column per cent).

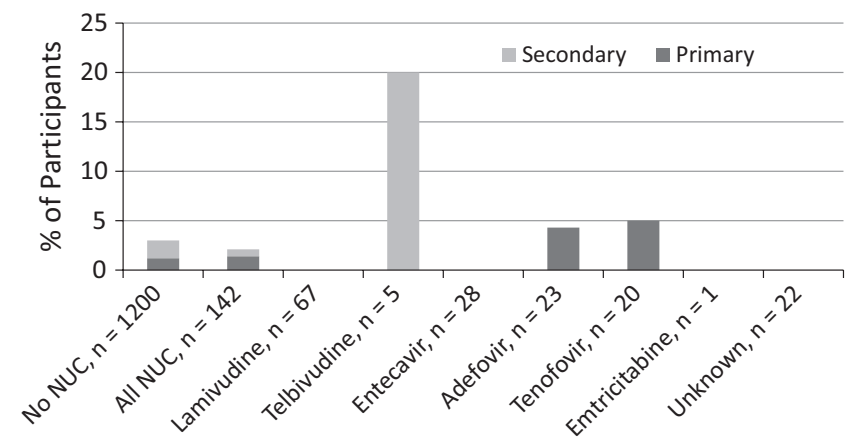

FIGURE 2 Prevalence of primary and secondary HBV-DR variants in relation to prior nucleos(t)ide analogues therapy

primary and secondary HBV-DR variants detected by Sanger sequencing by prior NUC therapy is shown in Figure 2.

\section{3 | Prevalence of primary and secondary HBV-DR variants based on NGS}

One hundred and ten participants with $>90$ days prior NUC exposure and available Sanger sequencing data and 110 participants with no prior NUC exposure matched for HBV genotype and baseline HBV DNA level were selected for NGS. Next generation sequencing data were obtained for 132/220 (60\%) participants: 66 with and 66 without prior NUC. Next generation sequencing data were not available for the other 88 participants, 44 in each group, due to insufficient sample or inadequate coverage of the region of interest. There were no differences in demographics, HBV genotype, HBV DNA level, ALT level and interval between stopping NUC and sample collection (in those who had prior NUC therapy) between the 132 participants with and 88 without NGS data (data not shown).

Characteristics of the 66 participants with and 66 participants without prior NUC exposure with NGS data were similar except that the group with prior NUC was more likely to be Asian $(P<.01)$ and to be younger $(P<.01)$ (Table 3). Among these 132 participants, NGS detected primary HBV-DR variants in 18 (13.6\%) participants: eight (12.1\%) with and 10 (15.2\%) without prior NUC therapy. In contrast, Sanger sequencing detected primary HBV-DR variants in four $(3.0 \%)$ participants: one (1.5\%) with and three $(4.5 \%)$ without prior NUC therapy. When detected using both methods, the primary HBV-DR variants detected by Sanger sequencing and NGS were concordant in three and discrepant in one participant (\#19) where NGS revealed an A181F variant that has not been confirmed to be associated with resistance to HBV NUC, while Sanger showed an A181V variant (Table 4).

Next generation sequencing confirmed the presence of secondary HBV-DR variants detected by Sanger sequencing in three participants and detected secondary HBV-DR variants in seven additional participants, making a total of 10 (7.6\%): six (9.1\%) with and four (6.1\%) without prior NUC therapy (Tables 3 and 4). The secondary HBV-DR variants detected by Sanger sequencing and NGS were concordant in all three participants. Only one (\#26) of the 10 participants with secondary HBV-DR variants was also found to have primary HBV-DR variant-M204V detected by NGS but not by Sanger sequencing.

Among the primary HBV-DR variants detected by NGS but not by Sanger sequencing, T184A/S was the most common variant observed, while V173L and L180M were the most common secondary HBV-DR variants detected by NGS but not by Sanger sequencing (Table 4).

In total, 28 of 132 (21\%) participants had either primary or secondary HBV-DR variants detected by NGS. Of these, eight (\#3, 4, 5, 7, 8, 10, 14 and 23) received NUC therapy after sample collection for HBV-DR variant testing. All eight received tenofovir, as monotherapy in six and in combination with emtricitabine in the other two participants. Median HBV DNA at the start of NUC treatment was $5 \log _{10} \mathrm{IU} / \mathrm{mL}$. HBV DNA decreased to levels below quantification in seven, with no breakthrough throughout the course of treatment (range 6.1-58.6 months); 


\begin{tabular}{|c|c|c|c|}
\hline Characteristics & $\begin{array}{l}\text { ALL } \\
N=132\end{array}$ & $\begin{array}{l}\text { Prior NUC } \\
\mathrm{N}=66\end{array}$ & $\begin{array}{l}\text { No NUC } \\
N=66\end{array}$ \\
\hline Age (y) & $39.5(32.8,48.3)$ & $37.3(30.9,44.1)$ & $42.0(33.6,52.5)$ \\
\hline Male & 49.2 & 54.5 & 43.9 \\
\hline \multicolumn{4}{|l|}{ Race } \\
\hline White & 4.6 & 4.5 & 4.6 \\
\hline Black & 8.4 & 1.5 & 15.4 \\
\hline Asian & 83.2 & 92.4 & 73.8 \\
\hline Other & 3.8 & 1.5 & 6.2 \\
\hline \multicolumn{4}{|l|}{ Place of birth } \\
\hline US and Canada & 13.6 & 9.1 & 18.2 \\
\hline $\begin{array}{l}\text { South America and } \\
\text { Other North America }\end{array}$ & 3.0 & 0.0 & 6.1 \\
\hline Europe & 0.8 & 1.5 & 0.0 \\
\hline Asia and Australia & 77.3 & 87.9 & 66.7 \\
\hline Africa & 5.3 & 1.5 & 9.1 \\
\hline \multicolumn{4}{|c|}{ Presumed source of hepatitis B virus (HBV) } \\
\hline Vertical transmission & 56.8 & 60.6 & 53.0 \\
\hline Horizontal Transmission & 21.2 & 24.2 & 18.2 \\
\hline Unknown & 22.0 & $15.2 \%$ & 28.8 \\
\hline $\begin{array}{l}\text { Hepatitis B e antigen } \\
\text { Positive }\end{array}$ & 25.8 & 28.6 & 23.1 \\
\hline HBV DNA $\left(\log _{10} I \mathrm{IU} / \mathrm{mL}\right)$ & $4.0(3.0,6.0)$ & $4.5(3.1,6.3)$ & $3.5(2.8,5.4)$ \\
\hline $\begin{array}{l}\text { Alanine aminotransferase } \\
x \text { ULN (U/L) }\end{array}$ & $1.4(1.0,2.1)$ & $1.5(1.0,2.1)$ & $1.35(1.0,2.0)$ \\
\hline \multicolumn{4}{|l|}{ HBV Genotype } \\
\hline A & 13.1 & 10.8 & 15.4 \\
\hline B & 36.9 & 40.0 & 33.8 \\
\hline C & 44.6 & 44.6 & 44.6 \\
\hline $\mathrm{D}$ & 4.6 & 4.6 & 4.6 \\
\hline Other & 0.8 & 0.0 & 1.5 \\
\hline \multicolumn{4}{|l|}{ Primary DR Variant } \\
\hline Sanger, n (\%) & $4(3.0)$ & $1(1.5)$ & $3(4.5)$ \\
\hline NGS, n (\%) & $18(13.6)$ & $8(12.1)$ & $10(15.2)$ \\
\hline \multicolumn{4}{|l|}{ Secondary DR Variant } \\
\hline Sanger, n (\%) & $3(2.3)$ & $2(3.0)$ & $1(1.5)$ \\
\hline NGS, n (\%) & $10(7.6)$ & $6(9.1)$ & $4(6.1)$ \\
\hline
\end{tabular}

ULN, upper limit of normal.

Unless otherwise noted, \% is shown for categorical variables. Median (25th percentile, 75th percentile) is presented for continuous variables.

All $P$ values $>$.05.

the remaining patient had decrease in HBV DNA from 6.3 to $1.4 \log _{10}$ $\mathrm{IU} / \mathrm{mL}$ after 6 months of treatment and was lost to follow-up.

\section{4 | DISCUSSION}

In this study of 1342 persons with chronic HBV infection living in the US and Canada, who were not receiving HBV NUC therapy
TABLE 3 Characteristics of participants with and without prior nucleos(t)ide analogues (NUC) therapy in subset of participants with next generation sequencing (NGS) 


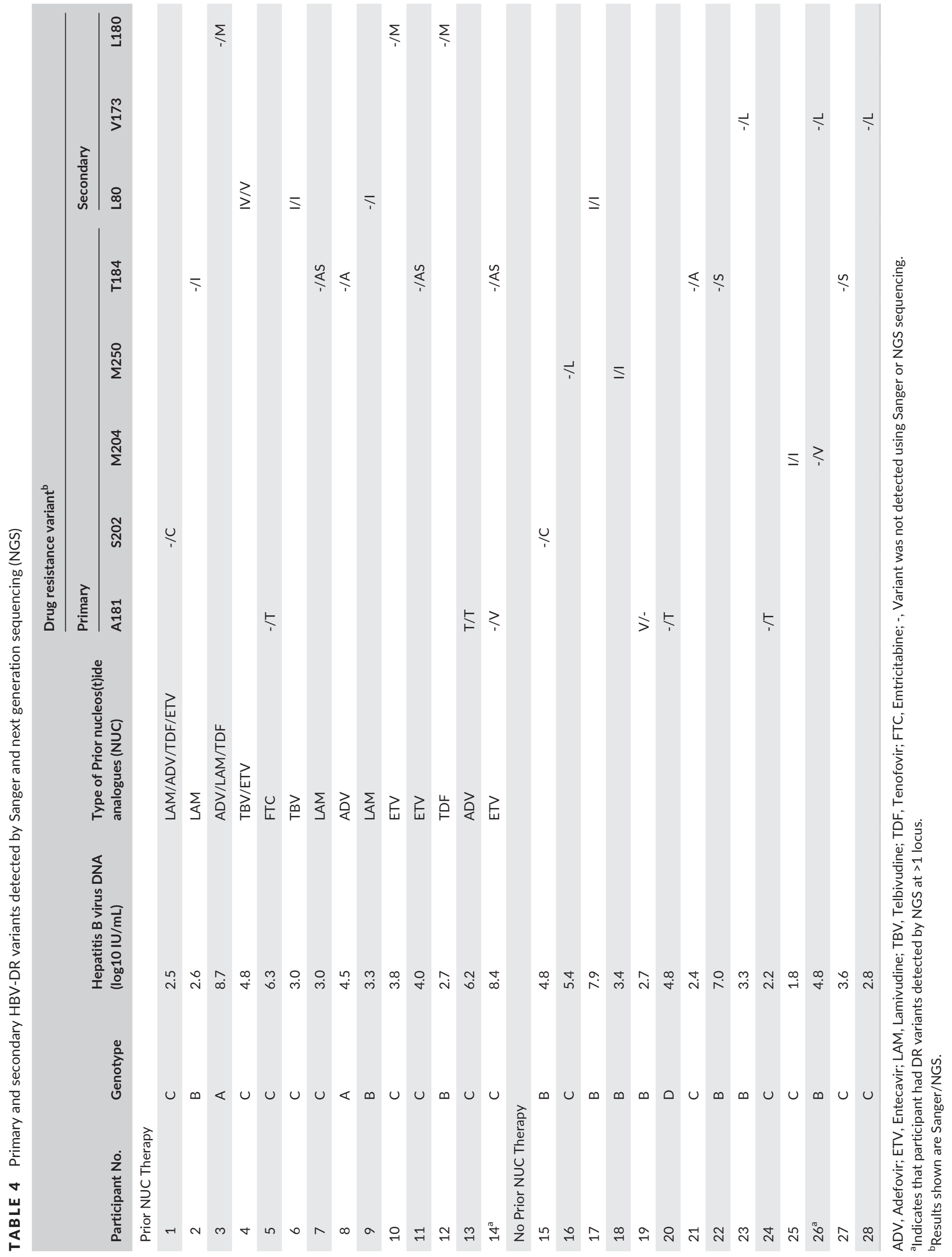


NUC therapy. The variants detected by Sanger sequencing and NGS were concordant except in one participant who had A181V by Sanger sequencing but $\mathrm{A} 181 \mathrm{~F}$ by NGS.

A systematic review and meta-analysis of the prevalence of HBV-DR variants from 106 studies involving 12212 patients with chronic HBV infection who had not received prior NUC therapy found that the global prevalence of all mutations (primary and secondary) was $5.7 \%, 5.4 \%$ for primary and $2.9 \%$ for secondary HBV-DR variants. ${ }^{6}$ There was significant geographical variation with the highest prevalence in China where the pooled prevalence rate of primary and secondary DR variants based on $>8000$ patients was $8 \%(95 \%$ $\mathrm{Cl}$ 6.63-9.38) while the pooled prevalence rate in other countries was $1.9 \%$ (95\% Cl 1.06-2.69). Much of the observed variability could be attributed to small number of patients or bias in selection of patients studied, uncertainty about prior exposure to NUCs, differences in sensitivities of the methods used for detection of HBV-DR variants, and differences in definitions of HBV-DR variants with some studies focusing on primary DR variants only while others including both primary and secondary DR variants, and yet others including polymerase gene variants of unclear significance. Globally, M204I/V was the most common primary HBV-DR variant detected with a pooled prevalence of $4.9 \%$, likely due to long-standing use of lamivudine as first-line therapy in many countries, particularly China. In our study, M250I/L/V was the most common primary HBV-DR variant detected by Sanger sequencing and $\mathrm{A} 181 \mathrm{~T} / \mathrm{V}$ and $\mathrm{T} 184 \mathrm{~A} / \mathrm{I} / \mathrm{S}$ were the most common primary HBV-DR variants in the subgroup with NGS data. The difference between results of our study and the meta-analysis is likely related to the early adoption of entecavir and tenofovir as first-line therapy in North America. Furthermore, among participants previously treated with lamivudine or adefovir, the interval between stopping these NUCs and DR variant testing was long-median 59 (range 3-165) months.

In our study, $69.3 \%$ of the participants were born in Asia and $8.9 \%$ in Africa, and likely acquired HBV infection in their home countries. Despite this, the prevalence of HBV-DR variants among participants in our study who had not received NUC therapy was substantially lower than that reported in the studies from Asia and Africa. This may be related to the fact that more than half of our participants born outside the US and Canada immigrated more than 10 years prior to enrolment into this study, which was before NUCs were in widespread use in Asia and Africa. Four other US studies that included 713 persons, predominantly Asians who had not received NUC therapy, had a similarly low prevalence of HBV-DR variants with a pooled prevalence of $0.71 \%$ (95\% Cl 0-1.34) based on Sanger sequencing or reverse hybridization. ${ }^{6,10-13}$ These findings support that HBV-DR variants are rarely found by Sanger sequencing among untreated persons with chronic HBV infection who reside in the US and Canada, and transmission of these variants to HBV-susceptible persons is uncommon. It should be noted that due to a high error rate during replication of HBV DNA, these variants may have emerged spontaneously and may not have been acquired at the time of infection. Although we found a higher prevalence of HBV-DR variants by NGS, the clinical significance of these variants present as a minor species is unclear, and most studies reported no impact of these minor variants on response to NUC therapy. ${ }^{14-16}$ Of the eight participants with DR variants detected by NGS who subsequently started NUC therapy, five had primary DR variants detected by NGS but none by Sanger sequencing. All eight had rapid virologic responses, and none had virologic breakthrough throughout the course of treatment.

HBV-DR variants selected during NUC therapy generally revert back to wild-type sequence after withdrawal of NUC therapy, although these variants may be archived and can be rapidly selected upon reexposure to the same NUC or other NUCs with cross resistance. In our study, we found that the prevalence of HBV-DR variants based on Sanger sequencing as well as NGS among participants with prior NUC was not higher than those without prior NUC, even though some had received NUC for more than 3 years. This may be related to the use of entecavir or tenofovir as first-line therapy in recent years, and the fact that majority of the treated participants had stopped NUC more than 6 months before testing. In addition, while NGS is more sensitive than Sanger in detecting minor variants, HBV-DR variants may represent such a low proportion of the virus pool after withdrawal of NUC that NGS may still fail to identify all cases.

Our study had a few limitations. The study population consisted of persons followed at tertiary care centres and may not be completely reflective of the general population with chronic HBV infection in the USA or Canada. Testing for DR variants with NGS was not possible in $40 \%$ of the samples, limiting the robustness of our conclusion on the prevalence of HBV-DR variants when tested by highly sensitive assays. However, the only difference between participants with NGS data and those in whom we had a technical failure with NGS was a shorter duration of prior NUC in those with NGS data, median 18.5 vs 25.1 months. All eight participants with baseline HBV-DR variants who subsequently received NUC therapy had rapid virologic responses with no breakthrough but the number is too small for us to ascertain the impact of these variants on response to NUC therapy. In clinical trials and in clinical practice, baseline testing for HBV-DR variants is not performed prior to initiating NUC therapy, and virological responses to NUC therapy-particularly with the newer NUCs entecavir or tenofovir-are achieved in the vast majority of patients.

In conclusion, our study of more than 1000 persons with chronic HBV infection residing in the US and Canada found a low prevalence of primary and secondary HBV-DR variants regardless of prior NUC therapy, supporting that these variants were either not present or disappear after withdrawal of NUC, and that transmission of these variants from patients receiving NUC to HBV-susceptible persons is probably rare. We confirmed that more sensitive assays such as NGS can detect HBV-DR variants in a higher proportion of persons with chronic HBV infection; however, the clinical significance of these variants present as minor species is uncertain and these variants may have emerged spontaneously. Our results could be related to the almost exclusive use of entecavir or tenofovir as first-line NUC therapy in North America in recent years and we support the World Health Organization recommendation that NUCs with high barriers to resistance should be available and accessible to all persons who need treatment for $\mathrm{CHB}$ worldwide. ${ }^{17}$ 


\section{DISCLOSURES}

ASL has received research grants from Bristol-Myers Squibb and Gilead. WML has received research grants from Bristol-Myers Squibb and Gilead. LGR, YKC, LP and HSL have no conflict to declare. The findings and conclusions in this report are those of the authors and do not necessarily represent the official position of the Centers for Disease Control and Prevention.

\section{ACKNOWLEDGEMENT}

In addition to the authors, the HBRN would like to acknowledge the contributions of the following: Harvard Consortium: Nezam Afdhal, MD, Asad Javaid, MBBS, Jianghe Niu, Johanna Han, Imad Nasser, MD (Beth Israel Deaconess Medical Center, Boston, MA, USA). Minnesota Alliance for Research in Chronic Hepatitis B: Alisha C. Stahler, Linda Stadheim, RN (Mayo Clinic Rochester, Rochester, MN, USA), Mohamed Hassan, MD (University of Minnesota, Minneapolis, MN, USA). Midwest Hepatitis B Consortium: Kathryn Rushing, RN, Rosemary A. Nagy, RDN, LD, MBA, Jacki Cerkoski, RN, MSN (Saint Louis University School of Medicine, St Louis, MO, USA), Debra DeMarco Shaw, RN, BSN, Lisa Kessels, RN, Michael K. Klebert, PhD, RN, ANP-BC (Washington University, St. Louis, MO, USA). University of Toronto Consortium: Seham Noureldin, PhD, Danie La, RN, Lucie Liu, MSc, CCRP, Diana Kaznowski, RN, Jiayun Chen, Doinita Vladutu, Orlando Cerocchi (Toronto Western \& General Hospitals, Toronto, Ontario). HBV CRN North Texas Consortium: Stacey Minshall, RN, BSN (Division of Digestive and Liver Diseases, University of Texas Southwestern Medical Center at Dallas, Dallas, Texas), Sheila Bass (University of Texas Southwestern, Dallas, TX, USA), Ethel Sauceda, BS (Baylor University Medical Center, Dallas, TX, USA). Los Angeles Hepatitis B Consortium: Samuel French, MD, Velma Peacock, RN (David Geffen School of Med, UCLA, Los Angeles, CA, USA). San Francisco Hepatitis B Research Group Consortium: Ashley Ungermann, MS, Claudia Ayala, MS, Emma Olson, BS, Ivy Lau, BS (University of California-San Francisco), Veronika Podolskaya, BS, NCPT, Nata DeVole, RN (California Pacific Medical Center, Research Institute). Michigan Hawaii Consortium: Barbara McKenna, MD, Kelly Oberhelman, PAC, Sravanthi Kaza, Bpharm, Cassandra Rodd, BS (University of Michigan, Ann Arbor, MI, USA), Leslie Huddleston, NP, Peter Poerzgen, PhD (The Queen's Medical Center, University of Hawaii, Honolulu, HI). Chapel Hill, NC Consortium: Jama M. Darling, M.D., A. Sidney Barritt, M.D., Tiffany Marsh, BA, Vikki Metheny, ANP, Danielle Cardona, PA-C (University of North Carolina at Chapel Hill, Chapel Hill, NC, USA). Virginia Commonwealth University Medical Center: Velimir A. Luketic, MD, Paula G Smith, RN, BSN, Charlotte Hofmann, RN (Virginia Commonwealth University Health System, Richmond, VA, USA). PNW/Alaska Clinical Center Consortium: Alycia Wolfstone, RN, MN (University of Washington Medical Center, Seattle, WA, USA) Jody Mooney, Lupita Cardona-Gonzalez (Virginia Mason Medical Center, Seattle, WA, USA). Liver Diseases Branch, NIDDK, NIH: Nancy Fryzek, RN, BSN, Elenita Rivera, BSN, Nevitt Morris, Vanessa Haynes-Williams. Liver Disease Research
Branch, NIDDK, NIH: Averell H. Sherker, MD, Rebecca J. Torrance, RN, MS, Sherry R. Hall, MS. Immunology Center: Mary E. Valiga, RN, Keith Torrey, BS, Danielle Levine, BS, James Keith, BS, Michael Betts, PhD (University of Pennsylvania, Philadelphia, PA, USA), Luis J. Montaner, DVM, DPhil (Wistar Institute, Philadelphia, PA, USA). Data Coordinating Center: Michelle Danielson, PhD, Tamara Haller, Geoffrey Johnson, MS, Stephanie Kelley, MS, Sharon Lawlor, MBA, Joan M. MacGregor, MS, Andrew Pelesko, BS, Donna Stoliker, Ella Zadorozny, MS (Graduate School of Public Health, University of Pittsburgh, Pittsburgh, PA, USA).

\section{REFERENCES}

1. Schweitzer A, Horn J, Mikolajczyk RT, Krause G, Ott JJ. Estimations of worldwide prevalence of chronic Hepatitis B virus infection: a systematic review of data published between 1965 and 2013. Lancet. 2015;386:1546-1555.

2. Zoulim F, Locarnini S. Hepatitis B virus resistance to nucleos(t)ide analogues. Gastroenterology. 2009;137:1593-1608. e1-2.

3. Lok AS, Zoulim F, Locarnini S, Bartholomeusz A, Ghany MG, Pawlotsky $J M$, et al. Antiviral drug-resistant HBV: standardization of nomenclature and assays and recommendations for management. Hepatology. 2007;46:254-265.

4. Ono SK, Kato N, Shiratori Y, Kato J, Goto T, Schinazi RF, et al. The polymerase L528M mutation cooperates with nucleotide binding-site mutations, increasing Hepatitis $B$ virus replication and drug resistance. J Clin Invest. 2001;107:449-455.

5. Teo CG, Locarnini SA. Potential threat of drug-resistant and vaccineescape HBV mutants to public health. Antivir Ther. 2010;15(3 Pt B): $445-449$

6. Zhang Q, Liao Y, Cai B, Li Y, Li L, Zhang J, et al. Incidence of natural resistance mutations in naive chronic hepatitis B patients: a systematic review and meta-analysis. J Gastroenterol Hepatol. 2015;30:252-261.

7. Lowe CF, Merrick L, Harrigan PR, Mazzulli T, Sherlock CH, Ritchie G. Implementation of next-generation sequencing for Hepatitis $\mathrm{B}$ virus resistance testing and genotyping in a clinical microbiology laboratory. J Clin Microbiol. 2016;54:127-133.

8. Ghany MG, Perrillo R, Li R, Belle SH, Janssen HL, Terrault NA, et al. Characteristics of adults in the hepatitis B research network in North America reflect their country of origin and Hepatitis B virus genotype. Clin Gastroenterol Hepatol. 2015;13:183-192.

9. Ganova-Raeva L, Ramachandran S, Honisch C, Forbi JC, Zhai X, Khudyakov Y. Robust Hepatitis B virus genotyping by mass spectrometry. J Clin Microbiol. 2010;48:4161-4168.

10. Nguyen MH, Garcia RT, Trinh HN, Nguyen HA, Nguyen KK, Nguyen $\mathrm{LH}$, et al. Prevalence of Hepatitis B virus DNA polymerase mutations in treatment-naive patients with chronic hepatitis B. Aliment Pharmacol Ther. 2009;30:1150-1158.

11. Margeridon-Thermet S, Shulman NS, Ahmed A, Shahriar R, Liu T, Wang $C$, et al. Ultra-deep pyrosequencing of Hepatitis B virus quasispecies from nucleoside and nucleotide reverse-transcriptase inhibitor (NRTI)-treated patients and NRTI-naive patients. J Infect Dis. 2009;199:1275-1285.

12. Vutien P, Trinh HN, Garcia RT, Nguyen HA, Levitt BS, Nguyen K, et al. Mutations in HBV DNA polymerase associated with nucleos(t) ide resistance are rare in treatment-naive patients. Clin Gastroenterol Hepatol. 2014;12:1363-1370.

13. Margeridon-Thermet S, Svarovskaia ES, Babrzadeh F, Martin R, Liu TF, Pacold $M$, et al. Low-level persistence of drug resistance mutations in Hepatitis B virus-infected subjects with a past history of Lamivudine treatment. Antimicrob Agents Chemother. 2013;57:343-349. 
14. Svarovskaia ES, Curtis M, Zhu Y, Borroto-Esoda K, Miller MD, Berg $\mathrm{T}$, et al. Hepatitis B virus wild-type and rtN236T populations show similar early HBV DNA decline in adefovir refractory patients on a tenofovir-based regimen. J Viral Hepat. 2013;20:131-140.

15. Liu Y, Fung S, Gane EJ, Dinh P, Flaherty JF, Svarovskaia ES, et al. Evaluation of HBV DNA decay kinetics in patients containing both rtM204V/I mutant and wild-type HBV subpopulations during tenofovir DF (TDF) monotherapy or combination therapy with emtricitabine (FTC)/TDF. J Med Virol. 2014;86:1473-1481.

16. Solmone M, Giombini E, Vincenti D, Rozera G, Testa A, Moscetti A, et al. Slow response to entecavir treatment in treatment-naive HBV patients is conditioned by immune response rather than by the presence or selection of refractory variants. Antivir Ther. 2014;19: 201-209.

\section{APPENDIX}

\section{The HBRN}

Harvard Consortium: Daryl T-Y Lau, MD, MPH (Beth Israel Deaconess Medical Center, Boston, MA, USA), Raymond T. Chung, MD (Massachusetts General Hospital, Boston MA, USA). Minnesota Alliance for Research in Chronic Hepatitis B Consortium: Lewis R. Roberts, MB, ChB, PhD (Mayo Clinic Rochester, Rochester, MN, USA), Coleman I. Smith, MD (University of Minnesota, Minneapolis, MN, USA). Midwest Hepatitis B Consortium: Adrian M. Di Bisceglie, MD, (Saint Louis University School of Medicine, St Louis, MO, USA), Mauricio LiskerMelman, MD (Washington University, St. Louis, MO, USA). University of Toronto Consortium: Harry L. A. Janssen, MD, PhD (Toronto Western \& General Hospitals, Toronto, Ontario), David K. Wong, MD (Toronto Western \& General Hospitals, Toronto, Ontario), Joshua Juan, MD (Toronto Western \& General Hospitals, Toronto, Ontario), Jordan Feld, MD, MPH (Toronto Western \& General Hospitals, Toronto, Ontario), Colina Yim (Toronto Western \& General Hospitals, Toronto, Ontario), Jenny Heathcote, MD (Toronto Western \& General Hospitals, Toronto, Ontario). HBV CRN North Texas Consortium: Robert Perrillo, MD, (Baylor University Medical Center, Dallas, TX, USA), Son Do, MD (University of Texas Southwestern, Dallas, TX, USA). Los Angeles Hepatitis B Consortium: Steven-Huy B. Han, MD (David Geffen School of Medicine, UCLA, Los Angeles, CA, USA), Tram T. Tran, MD (Cedars Sinai Medical Center, Los Angeles, CA, USA). San Francisco Hepatitis B Research Group Consortium: Norah A. Terrault, MD, MPH (University of CaliforniaSan Francisco), Mandana Khalili, MD, MAS (Department of Medicine, University of California- San Francisco, San Francisco, CA, USA),
17. WHO. Guidelines for the prevention, care and treatment of persons with chronic hepatitis B infection. 2015. http://www.who.int/hiv/pub/hepatitis/hepatitis-b-guidelines/en/. Accessed June 17, 2017.

How to cite this article: Lok AS, Ganova-Raeva L, Cloonan Y, et al. for the Hepatitis B Research Network (HBRN).

Prevalence of hepatitis $B$ antiviral drug resistance variants in North American patients with chronic hepatitis B not receiving antiviral treatment. J Viral Hepat. 2017;24:10321042. https://doi.org/10.1111/jvh.12732

Stewart L. Cooper, MD (California Pacific Medical Center, Research Institute \& Sutter Pacific Medical Foundation, Division of Hepatology, San Francisco, CA, USA). Michigan Hawaii Consortium: Robert J. Fontana, MD (University of Michigan, Ann Arbor, MI, USA), Naoky Tsai, MD (The Queen's Medical Center, University of Hawaii, Honolulu, HI). Chapel Hill, NC Consortium: Michael W. Fried, MD, (University of North Carolina at Chapel Hill, Chapel Hill, NC, USA), Keyur Patel, M.D. (Duke University Medical Center, Durham, NC, USA), Donna Evon, Ph.D. (University of North Carolina at Chapel Hill, Chapel Hill, NC, USA). PNW/Alaska Clinical Center Consortium: Robert C. Carithers, MD (University of Washington Medical Center, Seattle, WA, USA), Margaret Shuhart, M.D. (Harborview Medical Center, Seattle, WA, USA), Kris V. Kowdley, MD (Virginia Mason Medical Center, Seattle, WA, USA), Chia C. Wang, MD (Virginia Mason Medical Center, Seattle, WA, USA). Virginia Commonwealth University Medical Center: Richard K. Sterling, MD, MSc (Virginia Commonwealth University Health System, Richmond, VA, USA). Liver Diseases Branch, NIDDK: T. Jake Liang, MD (National Institutes of Health, Bethesda, MD). Liver Disease Research Branch, NIDDK: Jay H. Hoofnagle, MD (National Institutes of Health, Bethesda, MD), Edward Doo, MD (National Institutes of Health, Bethesda, MD). Immunology Center: Kyong-Mi Chang, MD, (University of Pennsylvania Perelman School of Medicine, Philadelphia, PA, USA), Jang-June Park, PhD (University of Pennsylvania Perelman School of Medicine, Philadelphia, PA, USA). Data Coordinating Center: Steven H. Belle, PhD, MScHyg (Graduate School of Public Health, University of Pittsburgh, Pittsburgh, PA, USA), Abdus Wahed, PhD (Graduate School of Public Health, University of Pittsburgh, Pittsburgh, PA, USA). Central Pathology: David Kleiner, MD, PhD. (Center for Cancer Research, National Cancer Institute, NIH, Bethesda, MD). 